\title{
INNOVATIONS IN PRIMARY CARE
}

\section{Humanwide: A Comprehensive Data Base for Precision Health in Primary Care}

\author{
Megan R. Maboney, MD' \\ Steven M.Asch, MD, MPH ${ }^{1,2}$ \\ 'Division of Primary Care and Population Health, Stanford Univer- \\ sity, Palo Alto, California \\ ${ }^{2}$ US Department of Veterans Affairs, Center for Innovation to \\ Implementation, Palo Alto, California
}

Ann Fam Med 2019;17:273. https://doi.org/10.1370/afm.2342.

\section{THE INNOVATION}

Stanford Primary Care launched Humanwide, a demonstration project to provide patients with genetic screening, wearable sensors, health assessment, and wellness coaching. We synthesized patient-level biometric, genetic, social, environmental, and behavioral data into the electronic health record for primary care teams' use in building individual care plans.

\section{WHO \& WHERE}

Humanwide is part of Stanford Medicine's larger threefold precision health vision - to predict and prevent disease more effectively, and to cure more precisely. ${ }^{1}$ Humanwide is the clinical implementation of precision health in a general primary care setting to identify high-risk patients in cancer previvor and preand early cardiovascular disease states for targeted, cost-effective treatments and interventions. We began with a 9-month design/thinking process, assessing patient and provider needs for comprehensive data and likely use cases in technology and genetics data-mediated care. From January to July of 2018, we recruited an initial group of 50 patients for proof of concept, taking care to include a diverse representation among ages, races/ethnicities, sexes, and medical complexities.

\section{HOW}

Humanwide entails: (1) an in-person wellness-oriented behavioral, social, and environmental health assessment; (2) Bluetooth-enabled home scales, glucometers, blood pressure cuffs, and pedometers; and (3) a genetic assessment for disease risk

Conflicts of interest: authors report none.

\section{Corresponding author}

Megan Mahoney, MD

Division of Primary Care and Population Health

Stanford University

211 Quarry Rd Ste 405, MC 5985

Palo Alto, CA 94304

mahoneym@stanford.edu and pharmacologic interactions. Digital health data from homebased and wearable devices connect to the electronic health record, are combined with genetic information, and are graphically displayed for health care teams to detect poor disease control early and prevent complications. Patient care activities include: (1) in-person and virtual wellness coaching; (2) referrals to community resources; and (3) care coordination among the primary care provider, the medical assistant, a certified health coach, a clinical pharmacist, a behavioral health practitioner, a nutritionist, a registered nurse, and a genetics counselor.

\section{LEARNINGS}

The genetics and digital health components of Humanwide are the most adaptable to general primary care. Enhanced education on evidence-based screening of genetic conditions among participating primary care patients and providers was helpful, in addition to the creation of an efficient referral process for genetics counseling. While the most common reason for enrollment was patient and provider interest in genetic testing, most patients availed themselves of a broad spectrum of Humanwide resources. Encouraging the use of wearable devices in a healthy population helped us identify multiple patients with early diabetes or hypertension, prompting early intervention and self-management. For example, in one patient the ambulatory blood pressure cuff detected intermittent high blood pressures later verified with a more formal 24-hour ambulatory blood pressure assessment. The patient was able to begin lifestyle modifications that allowed him to control his blood pressure. Pharmacogenetic testing enabled us to individualize the dosage and optimize the combination of medications based on a patient's drug-gene interactions. In one case we determined that a patient complaining of leg cramps was a slow metabolizer of statins, and we reduced his dose to resolve his cramps. Next steps include testing predictive analytics for interpretation of more complex data patterns.

Our early experience with Humanwide shows that creating a more comprehensive patient-centered data environment is feasible and acceptable to patients and providers. We hope to paint a much more comprehensive picture of patients' behaviors, genetics, and physical attributes than is commonly available in primary care today. Provider teams have just begun to determine how best to use these data to enhance care.

For additional information, including references, see it online at http://www.AnnFamMed.org/content/17/3/273.

Submitted August 15, 2018; submitted, revised, September 10, 2018; accepted October 3, 2018. 